
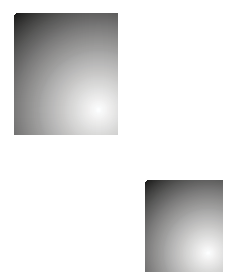

\title{
As Cinco Forças Amizade, Contato, Aprendizado, Cooperação e Competição como Campo de Força Estratégica de Redes: UM ESTUDO NO SETOR DE CALÇADOS E BOLSAS
}

\author{
The Five Forces Friendship, Contact, Learning, Cooperation and Competition \\ as Strategic Force Field networks: a study in shoes and bags industry
}

\author{
Carlos Alberto Gonçalves \\ Universidade FUMEC e Universidade Federal de Minas Gerais. Belo Horizonte, MG, Brasil.E-mail: carlos.iron@bol.com.br; carlos@ \\ face.ufmg.br;carlosag@fumec.br
}

Fernanda da Vitoria Lebarcky

Mestre em Adminsitração. Universidade Federal de Minas Gerais. Belo Horizonte, MG, Brasil. E-mail: fernandavl@ufmg.br

Cristiana Fernandes de Muylder

Doutora em Economia Aplicada pela UFV. Universidade FUMEC. Belo Horizonte, MG, Brasil. E-mail cristiana.muylder@fumec.br

\section{Resumo}

O presente trabalho apresenta um estudo de rede de relacionamento em um Arranjo Produtivo Local (APL). Foram estimadas as diferentes topologias de adensamento e a formação de constelações, como estratégia de aderência (ou repulsão) na rede APL em estudo, baseadas em Cinco Forças relacionais eleitas para o estudo, com base na literatura de estratégia de redes de relacionamento: Amizade, Contato, Aprendizado, Cooperação e Competição. O estudo foi feito a partir dos relacionamentos entre 42 organizações de um APL de calçados e bolsas. As estruturas de rede foram analisadas com o auxílio do software Ucinet 6 e NetDraw (BORGATTI, 2002). As redes com maior conectividade e menor fragmentação são as redes de Amizade e de Contatos, enquanto as redes com menor conectividade e maior fragmentação, respectivamente, são as redes de Competição, Cooperação e Aprendizado. Os resultados demonstram ainda que o grupo dos quatro maiores hubs da rede de aprendizagem é composto pelos dois maiores hubs da rede de Cooperação e pelos dois maiores hubs da rede de Competição.

Palavras-chaves: Cinco Forças. Redes. Arranjos Produtivos Locais. Cooperação. Competição.

\section{Abstract}

This work aim presents a study of relationship network in a Local Productive Arrangement (LPA). It has been evaluated the different topologies of consolidation and constellation formation as strategy of adherence (or repulsion) in the studied LPA, based on the relational Five Forces elected to the study based on strategy literature of relationship network: Friendship, Contact, Learning, Cooperation and Competition. The study has been created based on the relationship between 42 organizations from a LPA of Footwear and Handbags. The network structures have been analyzed with the assistant of the software Ucinet 6 and NetDraw (BORGATTI, 2002). The networks with higher connectivity and lower fragmentation are, respectively, the Competitive network, the Cooperation network and the Learning network. The results have also showed that the group of the four highest hubs in the learning network is composed of the two highest hubs of the Cooperation network and of the two highest hubs of the Competition network.

Keywords: Five Force. Network. Local Productive Arrangement. Cooperation. Competition. Learning. Five Forces. 


\section{INTRODUÇÃO}

As relações interorganizacionais são abordadas a partir de diversas perspectivas e áreas funcionais da Administração. Uma perspectiva emergente é a incorporação do conceito de redes sociais - conjunto de nós (pessoas ou organizações) ligados por relações sociais ou enlaces de tipos específicos (GRANOVETTER, 1976) - para a análise das trocas realizadas entre organizações. A noção de redes, utilizada inicialmente por uma sociologia relacional, vem sendo aplicada "[...] aos arranjos organizacionais flexíveis e plurais que se formam nos setores econômico, político ou social". (MIGUELETTO, 2001, p. 7)

Granovetter (2005) explica que a evolução da indústria e a estabilidade econômica das instituições ocorrem com o desenvolvimento de atividades estruturadas em torno de redes pessoais. Nesse sentido, a ideia de redes sociais agrega à teoria de estratégia a compreensão de que pessoas e organizações agem no interior de um sistema de relações concretas e em permanente desenvolvimento. Com a troca de informações sobre as operações (HITT et al., 2003), as empresas influenciam-se reciprocamente e contribuem para a melhoria dos resultados coletivos (GRIMM; SMITH, 1997). Dessa forma, a socialização entre organizações é parte de um processo importante para a aprendizagem organizacional (TAKEUCHI; NONAKA, 2008) e para a competitividade de um setor. (HUGGINS, 2009)

A fim de aprofundar a noção de que, no âmbito interorganizacional, os relacionamentos são relevantes para a aprendizagem e, portanto, para o desenvolvimento da indústria, este trabalho descreve e compara diferentes topologias em um arranjo produtivo local, a partir de uma abordagem de Análise de Redes Sociais (ARS).

Os agrupamentos de organizações competidoras em um mesmo setor, também tratados como "constelações" (GOMES-CASSERES, 1996), são mais ou menos aglutinados pela combinação de diferentes intensidades relacionais. $\mathrm{O}$ artigo apresenta Cinco Forças de adensamento - Amizade, Contato, Aprendizado, Cooperação e Competição - que configuram a intensidade de adensamentos topológicos em constelações distintas nos arranjos produtivos locais. No presente trabalho entende-se que os fatores "Meta-Categóricos" constituem o que se passará a chamar de campos de forças atrativas que fazem os atores "gravitarem" entre si, estabelecer atração e ou repulsão, produzindo configurações topológicas diferenciadas, enfim, criando maior adensamento ou dispersão nas redes de APL.

Considera-se Arranjo Produtivo Local (APL) uma aglomeração de empresas, localizada em um mesmo território, que apresenta especialização produtiva $e$ mantém algum vínculo, ainda que incipiente, de articulação, de interação, de Cooperação e de aprendizagem entre si e com outros atores locais, tais como: governo, associações empresariais, instituições de crédito $e$ instituições de ensino e pesquisa. (CASSIOLATO; LASTRES, 2003)

A propensão ao relacionamento pela proximidade geográfica, aliada à expectativa governamental quanto ao potencial de fomento para o desenvolvimento das estruturas de APLs, torna tais aglomerações objetos frutíferos para estudos sobre as formas de ligação, relacionamentos e aprendizagem, relevantes para a esfera científica, a pragmática das relações, política públicas de fomento. Além disso, APLs ilustram a simultânea dinâmica da Cooperação e da Competição em redes interorganizacionais, fenômeno que necessita de maior aprofundamento na literatura de redes sociais. (VENKATRAMAN; LEE, 2004)

\section{Referencial Teórico}

A seção de referencial teórico expõe conceitos e medidas da Análise de Redes Sociais (ARS) e apresenta considerações sobre a imersão social de organizações em redes de relacionamentos.

\subsection{Análise de Redes Sociais}

A metodologia de análise de redes tem apresentado contribuições importantes para as áreas de Saúde, Sociologia, Psicologia, Administração Pública, Física, Estratégia, Comportamento Organizacional, Comunicação e Ciências da Computação (PROVAN; FISH; SYDOW, 2007). O Brasil possui menor tradição na utilização da metodologia na área da Administração, em relação à produção anglo-saxônica. (MARTES et al., 2006)

Em uma rede social, segundo Recuero (2004), as pessoas são os nós e as arestas são os laços gerados por meio da interação social. Um laço, ou relação entre 
dois atores, tem força [strength] e conteúdo. A força refere-se à intensidade da relação e o conteúdo refere-se ao tipo de informação ou interesses compartilhados nos relacionamentos. Granovetter (1976) define que a força de um laço envolve a combinação da quantidade de tempo, da intensidade emocional, da intimidade (que espelha Amizade), confiança mútua, e dos serviços recíprocos que caracterizam o laço. Nesse sentido, um laço pode ser forte, fraco ou ausente (quando há falta de qualquer relacionamento). Diferentes conteúdos relacionais podem ser compreendidos como distintas forças de adensamento, que geram estruturas distintas.

Os principais tipos de informação em análise de redes são informações de atributo, como atitudes, comportamentos, qualidades e características, denominadas também "propriedades", e informações relacionais, como Contatos, laços e conexões que relacionam um agente a outro (SCOTT, 1991). A representação gráfica da estrutura social e das propriedades dos nós é conhecida como grafos ou sociogramas. (FREEMAN, 2004)

A partir desses conceitos, a abordagem estrutural aplicada aos estudos de redes sociais experimentou particular desenvolvimento metodológico. Estudos com essa perspectiva, conhecida como análise de redes sociais (ARS), tendem a explicar o comportamento com base na rede de relacionamentos. Nesse sentido, o comportamento de cada ator é interpretado a partir de padrões de vínculos interacionais e morfológicos em sua rede social. Uma vez que os dados necessários são coletados, o analista é então confrontado com o problema da descoberta de regularidades no material. (MITCHELL, 1974)

Em relação às medidas desenvolvidas para a análise de redes, "densidade" e "centralidade", elas, em particular, tornaram-se básicas para a análise. A densidade representa a razão entre as relações observadas e o número teórico possível de relações - dado por $\mathrm{N}(\mathrm{N}$ - 1)/2, sendo $\mathrm{N}$ o número de nós. Em pequenos grupos, a densidade é usualmente tratada como uma medida de coesão do grupo e como indicação parcial da extensão em que um grupo é primário ou fechado. (SOUZA; QUANDT, 2008)

A centralidade indica o quanto um ator central interliga diversos outros atores não conectados entre si. É calculada com base na quantidade de elos que se colocam entre eles. Mensurar a centralidade de um ator significa identificar a posição em que ele se encontra em relação à comunicação e às trocas na rede (MARTELETO, 2001). O número de enlaces que chega a um ator na rede representa a medida de centralidade indegree e o número de enlaces que parte de um ator para outros representa a medida de centralidade outdegree (BORGATTI; CARLEY; KRACKHARDT, 2006). A quantidade de enlaces totais de um nó contribui para o grau de centralidade geral (degree), medida que representa a importância de um ator para a troca de informações na rede. A combinação dessas centralidades auxilia a formação do conceito de "Hubs" - nós mais centrais com alta conectividade. Em tecnologia da informação, hubs são sites da web que contêm links para os sites mais acessados. (BORGATTI, 2002)

Destaca-se ainda a estruturação do conceito de "cliques" em ARS. O conceito reflete o reconhecimento da importância da formação de subgrupos na rede grupos de atores em que cada um é diretamente e fortemente conectado a todos os outros, como sub-redes de atores com relações mais estreitas. (EMIRBAYER; GOODWIN, 1994)

\subsection{Relacionamentos Interorganizacionais}

Os membros da rede interorganizacional podem estar conectados por inúmeros tipos de conexões e fluxos, como informação, material, recursos financeiros, serviços e suporte social (PROVAN; FISH; SYDOW, 2007). O conteúdo das relações entre os nós é limitado pela concepção do pesquisador. (BRASS et al., 2004)

Segundo Oliver (1990, p. 241), as relações interorganizacionais são demarcadas por "[...] transações relativamente constantes, fluxos e ligações que ocorrem entre uma ou mais organizações em seus ambientes". Em geral, são definidas por conexões entre empresas (CASTELLS, 1999), estabelecidas por um grupo de atores que se articulam para conduzir ações de forma Cooperada (construção de vínculos Cooperativos) e que partilham uma visão produzida da realidade. (MIGUELETTO, 2001)

A rede interorganizacional formada por um grupo de competidores de setores correspondentes ou similares é também tratada como "constelações" (GOMES-CASSERES, 1996). As constelações, explícitas ou implícitas (LAZZARINI, 2004), são estruturadas a partir de relacionamentos horizontais entre organizações, que se aproximam com intensidades distintas. 
As organizações fazem parte de um conjunto complexo de relacionamentos (redes), mecanismos importantes para o processo de institucionalização de práticas entre organizações. Para reforçar essa ideia, Marsden (1981) resgata o conceito de imersão social [embeddedness], que se refere ao fato de que trocas e discussões dentro de grupos têm tipicamente uma história de estabilização de vínculos entre os membros. O foco estrutural da imersão social está no papel da organização em função da posição ocupada na estrutura da rede (OWEN-SMITH; POWELL, 2007). A partir dessa ideia, Giuliane (2007, p. 142), ao considerar clusters empresas espacialmente aglomeradas em uma mesma indústria, defende que "[...] relacionamentos de mercado e relacionamentos sócio-institucionais são considerados veículos importantes de difusão de conhecimento em nível intracluster". Por causa da posição ocupada na rede, a autora explica que "[...] algumas firmas serão mais centrais do que outras [...]" nesse processo. (GIULIANE, 2007, p. 145)

Portanto, sugere-se que a estrutura da rede possui um efeito no Aprendizado social e que as informações oriundas da rede possuem substancial influência na organização (Kraatz, 1998) e no desempenho de todo o sistema. (LIPPARINI; LOMI, 1999)

Em relação à correspondência entre posicionamento na rede e desempenho, Powell, Koput e Smith-Doerr (1996) encontraram em seus trabalhos uma tendência de desconectividade, na qual organizações mais antigas e com menos vínculos são mais suscetíveis a errarem. Múltiplas conexões e centralidade significam recebimento de mais informação da rede e, consequentemente, impacto positivo em desempenho (BORGATTI; LI, 2009), em processos de inovação (TSAI, 2001) e na acumulação de experiências e habilidades. (GIULIANE; PIETROBELLI, 2011)

No processo de concorrência, as empresas influenciam-se reciprocamente e contribuem para a melhoria mútua da Competitividade (GRIMM; SMITH, 1997) quando trocam informações sobre suas operações (HITT et al., 2003). Tal melhoria da vantagem Competitiva por meio de relacionamentos advém da criação de conhecimento no nível da rede interorganizacional. (AHMADJIAN, 2008)

Nesse sentido, Huggins (2009) argumenta que a competitividade de uma empresa ou de um setor é, cada vez mais, uma função do valor criado por meio dos fluxos interorganizacionais de Conhecimento $e$ Aprendizado. Essa constatação adquire relevância peculiar no contexto das aglomerações produtivas e da sinergia local existente em APLs.

O processo de criação de conhecimento na rede acontece quase da mesma forma que no interior da empresa. Sua intensidade dependerá da estrutura de compartilhamento de informações. De acordo com o modelo SECI, de Takeuchi e Nonaka (2008), também aplicável ao Conhecimento no nível da rede, o Conhecimento tácito é criado por meio da socialização, convertido de tácito para explícito por meio da externalização, recombinado com outras formas de conhecimento explícito e novamente revertido em conhecimento tácito por meio da internalização. A socialização entre membros de distintas organizações é, portanto, parte de um processo importante para a aprendizagem organizacional e para a competitividade da indústria. Os relacionamentos estabelecidos, contudo, devem alcançar um equilibrio de interesses individuais e coletivos.

Em relações horizontais dentro de um setor, empresas concorrentes precisam exibir forte postura Cooperativa para colher os benefícios da colaboração e dispor de forte senso competitivo para garantir que os outros não bloqueiem seus interesses. Isto é, as organizações "[...] precisam estar imersas [embedded] em relações cooperativas na rede e, ao mesmo tempo, independentes o suficiente para manipular o poder em seu próprio benefício". (WIT; MEYER, 2010, p. 379)

\section{Método Aplicado}

Esta seção apresenta informações sobre o caso estudado, principais procedimentos de coleta e análise dos dados.

\subsection{O Estudo de Caso}

Foi desenvolvido um estudo de caso no APL de Calçados e Bolsas da Região Metropolitana de Belo Horizonte (RMBH). O polo é especializado na produção de artigos femininos e de couro, principal matéria-prima utilizada. De acordo com a FIEMG (2011), o setor de calçados e bolsas da RMBH é conhecido atualmente pela grande concentração de profissionais talentosos e pelo desenvolvimento de produtos de 
alto valor agregado. Cerca de $50 \%$ das empresas são familiares, $69,1 \%$ são micro, $23,6 \%$ são pequenas $e$ $7,3 \%$ são médias.

Nos últimos anos, as empresas do setor de calçados e bolsas vêm enfrentando séria crise devido à forte concorrência de produtos chineses, o que culmina na retração gradativa do setor. Segundo dados do RAIS/ MTE Brasil (2009), de 2008 para 2009 houve uma redução de $28 \%$ no número de empresas e de $30 \%$ no número de empregos. Nesse contexto, a mobilização do SINDIBOLSAS e do SINDICALÇADOS para a formalização do polo como um APL, em 2007, foi uma tentativa de aproveitar a identidade empresarial local com a moda e de minimizar o impacto da retração do setor.

\subsection{A Coleta de Dados}

A amostra de redes é, por natureza, independente e não probabilística. Em geral, os pesquisadores de redes estão mais focados na descoberta de relações entre parâmetros/variáveis e a base teórica do que em deduzir padrões aplicáveis a uma população de redes alheia ao objeto de estudo. (SOUZA; QUANDT, 2008)

A seleção de empresas do APL para comporem a rede de empresas do estudo obedeceu a critérios de participação nas atividades do sindicato, participação nas oficinas do Programa de Desenvolvimento de APLs e importância da empresa para a economia local. A partir desses critérios, quatro especialistas do setor apontaram 40 empresas. Dentre as 40 empresas componentes da rede, cinco desativaram as operações e/ou não foram localizadas nos telefones e endereços disponíveis e 13 recusaram-se responder às entrevistas e preencher os questionários. Assim, a coleta de dados do estudo envolveu a realização de entrevistas e aplicação de questionários com quatro especialistas e com 22 proprietários das empresas previamente selecionadas, sendo 20 fábricas e dois fornecedores, no período de agosto a dezembro de 2012. O Sebrae e o Sindicato também foram incluídos na rede, o que resultou em uma constelação com 42 organizações (nós da rede).

As seguintes empresas foram inseridas e analisadas na rede são a: Voot, Veratro, Uzee bolsas, Trapézio, Thomaz Rabelo, TBO, Spatifilus, Solalex, Silvya Brandão, Schmuck, RRV, Rogério Lima, Príncipe Verde, Paula Bahia, North Pak, Minas Pre, Marcia Macedo,
Lusis e Atos, Luiza Barcelos, La Spezia, Kasttidade, Junia Gomes, Gruller, Evidência, Eduardo Pires, Doiss, Debora Germani, Dasa, Covenant, Celso Afonso, Casual, Calçare, Bruno Cross, Bl Baby, Bazze, Banana Bag, Balaia, Arzon, Andrezza Dias e Adô. A fim de preservar os dados individuais das empresas, para a descrição dos resultados nomes fictícios extraídos da lista de planetas e estrelas mais brilhantes foram aleatoriamente atribuídos às empresas componentes da rede.

Os questionários foram desenvolvidos com a abordagem de lista fixa para cada conteúdo relacional, com escalas de relações ordinais totais (full-rank ordinal measures of relations).

\subsection{A Análise e Tratamento dos Dados}

As entrevistas foram gravadas e, posteriormente, transcritas. O conteúdo das entrevistas foi submetido à análise de conteúdo (BARDIN, 1977). O conteúdo dos questionários com os atributos das organizações e com as interações realizadas foi tabulado em Excel e, em seguida, importado para o software Ucinet. O Ucinet, apesar da não gratuidade, é uma das ferramentas mais populares para a análise de redes, devido à variedade de medidas que consegue processar. (MELO; RÉGIS, 2012)

O processamento dos dados no Ucinet foi realizado com parâmetros de laços direcionais (importa a direção do enlace, quem emite e quem recebe o enlace), mínimo de três nós para a caracterização de cliques ( $\mathrm{p}=3)$, introdução de 42 nós $(\mathrm{N}=42)$ e conjunto de cinco relações sociais $(r=5)$, consideradas como forças de adensamento. O conjunto das relações sociais pode ser descrito como: $\mathrm{R}=\{$ Amizade (ou laços pessoais), Contato, aprendizagem, Cooperação, Competição\}. As medidas descritas comparativamente neste trabalho, produzidas pelo Ucinet, são: conectividade, fragmentação, número de enlaces, número de cliques e densidade. Além disso, comparam-se os componentes da estrutura central (centralidade indegree) e os maiores hubs de cada topologia.

Posteriormente, sociogramas foram elaborados com o auxílio do Netdraw para apoiar a visualização e a análise do conjunto de relações. O Netdraw é uma ferramenta acoplada ao Ucinet, desenvolvida pelos mesmos autores, com a finalidade de permitir a organização gráfica dos dados em formato de redes. 
Durante as descrições das estruturas das redes, o relacionamento entre os proprietários das empresas é também tratado como relacionamento entre empresas, enlaces ou laços. E os respondentes do questionário são tratados como proprietários, empresas, organizações, nós ou nodos.

\section{Apresentação e Discussão dos Resultados}

A subseção de apresentação dos resultados divide-se em: (1) apresentação das topologias de Amizade (ou de laço pessoal), Contatos, Aprendizagem, Cooperação e Competição em sociogramas e (2) comparativo das medidas de ARS. Na subseção de discussão, são desenvolvidas algumas reflexões sobre os resultados encontrados.

\subsection{Apresentação das Forças e Topologias condicionadas de Redes}

Ao longo da apresentação dos dados, as empresas de calçados são denominadas com nomes fictícios para evitar suas identificações, como: Aldebaram, Alpha, Antares, Athena, Eris, Haumea, Kaos, Marte, Mimosa, Neturno, Numki, Polaris, Rigel, Saturno, Shaula, Sírius, Spica, Terra e Urano. Por sua vez, os fornecedores são referidos como F1, F2 e F3 e os fabricantes de bolsas são denominados: Sargas, Júpiter, Plutão, Bellatrix, Polux, Castor, Altair, Acrux, Vênus, Atria, Hamal, Peacock, Deneb, Kaitos, Mirfak, Mercúrio, Ceres e Makemake.

\subsubsection{A Topologia da Rede (Força de) Amizade}

O sócio dirigente de cada empresa participante da pesquisa indicou com quais outros proprietários de empresas do setor possuía relacionamentos pessoais (Amizade ou laços familiares). Dentre as 42 organizações que constavam na lista, 37 mencionaram ou foram mencionadas em relações de Amizade. As cinco empresas que não possuem laços pessoais com outras do setor - Shaula, Altair, Kaus, Peacook e Mirfak - estão representadas de forma isolada no sociograma (Figura 1). Apesar de haver Amizade entre proprietários de empresas que fabricam produtos diferentes (bolsas e calçados), há uma tendência ao estabelecimento de laços de Amizade com empresas que fabricam o mesmo produto. Em outras palavras, pertencer ao mesmo negócio facilita o início ou manutenção de vínculo de Amizade.

A Figura 1 apresenta a rede de Amizade com nodos dimensionados pelo número de enlaces (medida centralidade degree do Ucinet). A medida aponta a importância relativa de cada nó para os fluxos da rede.

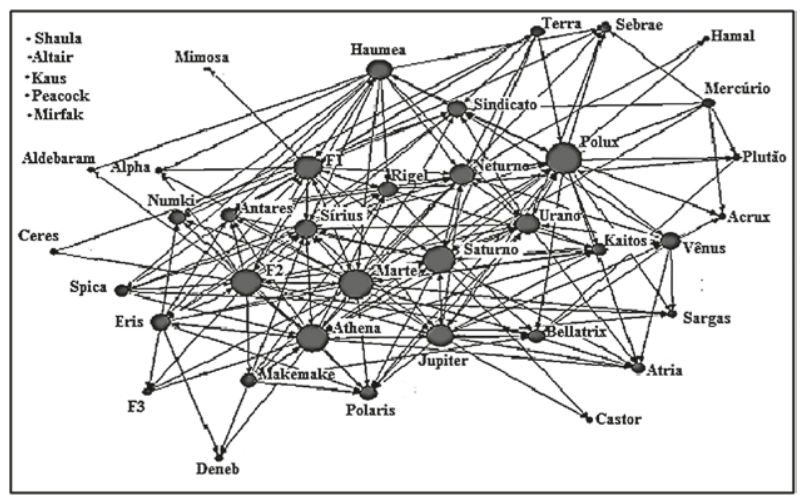

Figura 1: Laços de Amizade

Fonte: Dados primários

A constelação da força Amizade possui as seguintes medidas: enlaces 211 , densidade $12,3 \%$, 8 cliques, fragmentação 0,623 e conectividade 0,377. Nota-se elevado adensamento pela densidade e conectividade.

\subsubsection{A Topologia da Rede (Força de) Contatos}

De maneira análoga, a rede de Contatos indica o fluxo de interação entre os empresários, por telefone, e-mail ou pessoalmente. Dos 42 nodos presentes na rede, quatro não indicaram e não foram indicados por qualquer outro: Peacock, Kaus, Shaula e Mirfak. A similaridade em produto fabricado também é importante para o estabelecimento de Contato entre as empresas, já que tendem a se localizar na mesma zona do grafo. A Figura 2 apresenta a rede de Contatos com nodos dimensionados pelo número de enlaces (medida centralidade degree do Ucinet). 


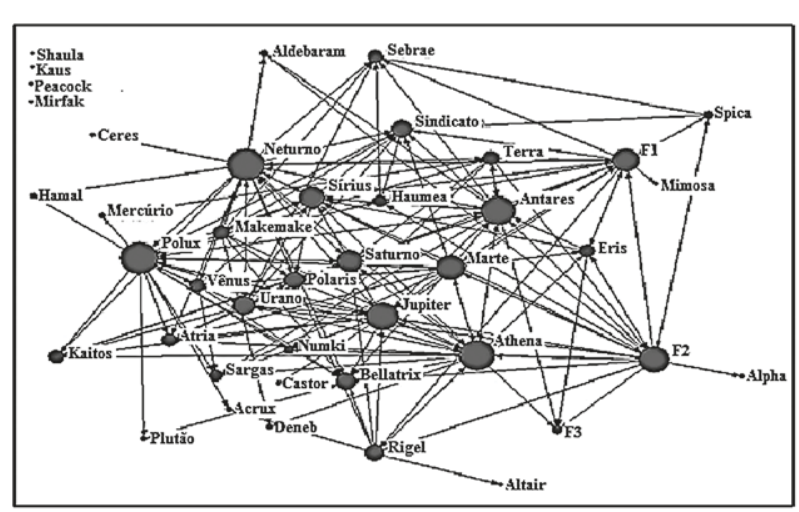

Figura 2: Laços de Contatos

Fonte: Dados primários

A constelação da força Contatos possui as seguintes medidas: enlaces 194, densidade $11,3 \%, 8$ cliques, fragmentação 0,623 e conectividade 0,377. Nota-se elevado adensamento da rede de Contatos pela densidade e conectividade.

\subsubsection{A Topologia da Rede (Força de) Aprendizagem}

A rede de aprendizagem apresenta quais empresas/empresários aprendem sobre o negócio com quais outras empresas/empresários do setor. A Figura 3 ilustra a rede de aprendizagem com nodos dimensionados pela centralidade degree.

Das 42 empresas da rede, 12 não aprendem com qualquer outra empresa e não são referência em aprendizagem para ninguém, constituindo-se como nós isolados na rede. Identificou-se que todas as empresas com faturamento anual acima de 1,1 milhão aprendem ou ensinam na rede (estão conectadas a outros nós). Ademais, empresas de calçados e bolsas aprendem umas com as outras.

A constelação da força aprendizagem possui medidas: enlaces 81 , densidade 4,7\%, 0 cliques, fragmentação 0,870 e conectividade 0,130 . Nota-se falta de subgrupos com enlaces recíprocos (cliques), rede mais fragmentada e menos densa.

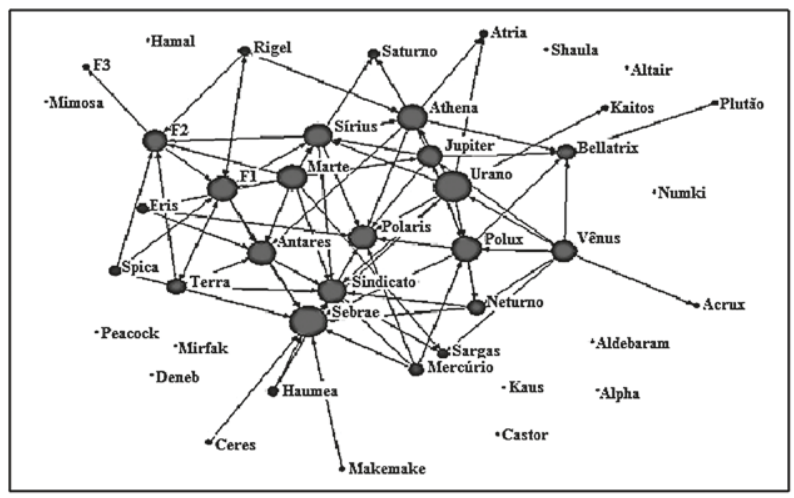

Figura 3: Laços de aprendizagem

Fonte: Dados primários

\subsubsection{A Topologia da Rede (Força de) Cooperação}

As empresas apontaram com quais outras mantinham ações de colaboração mútua, podendo ser representadas por ações pontuais (pesquisas conjuntas, contratação de consultores, organização de eventos para publicidade do setor, etc.) ou por parcerias em processos (compra de matéria-prima, produção, desenvolvimento de novos produtos, etc.). A Figura 4 apresenta a rede de Cooperação com nodos dimensionados pelo número de enlaces (medida centralidade degree do Ucinet).

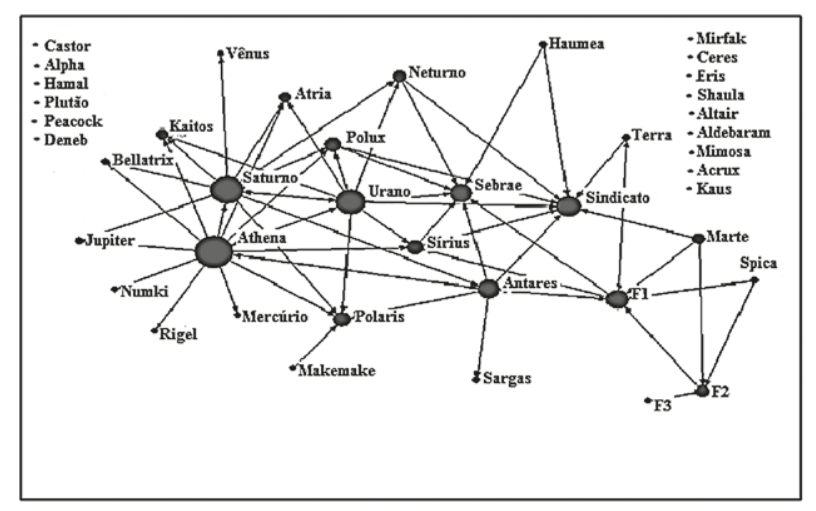

Figura 4: Laços de Cooperação

Fonte: Dados primários

Observa-se que 15 empresas da rede estão isoladas - não mencionaram ou não foram mencionadas em relações de Cooperação. Curiosamente, as empresas fabricantes de bolsas não possuem ações colaborativas conjuntas entre si. 
A constelação da força Cooperação possui medidas: enlaces 61 , densidade 3,5\%, 0 cliques, fragmentação 0,886 e conectividade 0,114 . Nota-se falta de subgrupos com enlaces recíprocos (cliques), rede ainda mais fragmentada e menos densa.

\subsubsection{A Topologia da Rede (Força de) Competição}

As empresas do setor de calçados e bolsas foram questionadas sobre quem consideravam seus concorrentes.

Algumas empresas de calçados (Neturno e Polaris) são apontadas por empresas de bolsas e algumas empresas de bolsas (Atria, Vênus e Acrux) são apontadas por empresas de calçados. Isso acontece porque tais empresas fabricam os dois produtos (bolsas e calçados).

A Figura 5 apresenta a rede de Competição com nodos dimensionados pelo número de enlaces (medida centralidade degree do Ucinet).

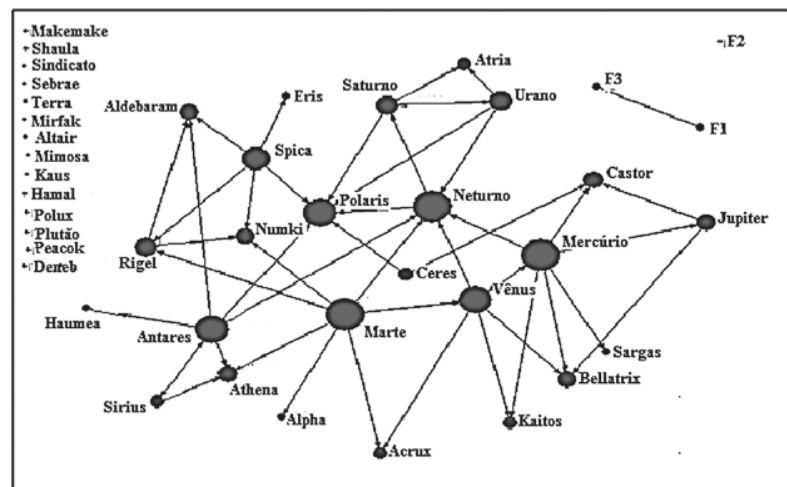

Figura 5: Laços de Competição

Fonte: Dados primários

A constelação da força Competição possui medidas: enlaces 48, densidade 2,8\%, 0 cliques, fragmentação 0,951 e conectividade 0,037 . Observa-se, pelas medidas associadas, que a força Competição é a que menos adensa organizações na constelação.

\subsection{Apresentação das Medidas de Redes}

A Tabela 1 apresenta os valores em conjunto das Cinco Forças relacionais para análise comparativa. Observa-se que a força Amizade apresenta os melhores indicadores relativos à agregação na rede, em relação às demais.

Tabela 1: Medidas comparativas das Cinco Forças de adensamento

\begin{tabular}{|c|c|c|c|c|c|}
\hline 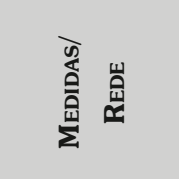 & & ن & 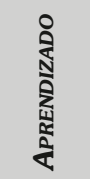 & 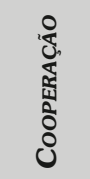 & 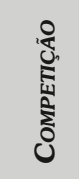 \\
\hline Enlaces & 211 & 194 & 81 & 61 & 48 \\
\hline Densidade & $12,3 \%$ & $11,3 \%$ & $4,7 \%$ & $3,5 \%$ & $2,8 \%$ \\
\hline Cliques & 8 & 8 & 0 & 0 & 0 \\
\hline Fragmentação & 0,623 & 0,623 & 0,870 & 0,886 & 0,951 \\
\hline Conectividade & 0,377 & 0,377 & 0,130 & 0,114 & 0,037 \\
\hline
\end{tabular}

Fonte: Dados primários

Observa-se na Tabela 1 que as redes mais densas são a de Amizade (12,3\%) e a de Contatos (11,3\%). As relações pessoais são, portanto, responsáveis pelas organizações serem mais conectadas entre si do que relações de parceria nos negócios, Aprendizado ou Competição. Os fluxos de aprendizagem, contudo, conectam mais a rede do que relações de Cooperação ou Competição. As redes com maior conectividade e menor fragmentação são as redes de Amizade e de Contatos, enquanto as redes com menor conectividade e maior fragmentação, respectivamente, são as redes de Competição, Cooperação e Aprendizado. Consistentes com a medida de densidade, as medidas de conectividade e de fragmentação indicam que, em redes de Amizade e de Contatos, as informações são capazes de percorrem distâncias maiores e alcançarem um número maior de organizações.

O número de relações presentes na rede de Aprendizado (81 enlaces) representa $42 \%$ do número de relações da rede de Contatos (194 enlaces). Como apontou a análise das entrevistas, o Aprendizado que advém de empresas concorrentes ou de instituições que apoiam o setor (Sebrae e Sindicato Local de Calçados e Bolsas) é transmitido pela interação direta ou pela observação dos produtos e práticas no mercado. Ou seja, os enlaces de Aprendizado são em determinada parcela consequência de enlaces de Contato e em outra parcela consequência da observação no mercado. 
Visto isso, identifica-se que grande parte das interações realizadas pelos proprietários das empresas distribui informações irrelevantes para o Aprendizado organizacional. Os vínculos pessoais também figuram como não significativos para a transmissão de aprendizagem no setor.

Subgrupos fortemente conectados (cliques) apenas foram identificados nas redes de Amizade $e$ de Contatos. Assim, reforça-se que a forte conexão existente em relações pessoais e em interação não determina mútuo Aprendizado.

Os nós mais referenciados da rede (maior centralidade indegree) ocupam posição mais central dos sociogramas e determinam a estrutura de influência em cada topologia. As organizações com maiores graus de centralidade indegree na rede de Amizade são: Sírius (17), Neturno (16), Saturno (15), Polux (14), Sindicato (14) e Antares (13). Na rede de Contatos, os maiores graus de centralidade indegree são das organizações: F1 (22), Sindicato (21), Sírius (14), Antares (14), Saturno (12), Polux (12) e Neturno (12). Na rede de Aprendizado, a medida é maior dentre: Sebrae (23), Sindicato (15), Polaris (13), Bellatrix (10), Polux (8), Antares (7), Sargas (7) e F2 (7). A rede de Cooperação possui como maiores graus de centralidade indegree: Sindicato (17), Sebrae (15), F1 (9), Polaris (5), Antares (5) e Sirius (4). E, por fim, a rede de Competição possui as maiores medidas com as organizações: Polaris (13), Neturno (11), Bellatrix (8), Castor (8) e Numki (7).

O Quadro 1 reúne as 32 posições de maior prestígio (respectivamente mais citadas de cada rede), ocupadas por um número de 14 organizações. São as organizações que mais polarizam a aglutinação da constelação.

\begin{tabular}{|c|c|c|c|c|}
\hline 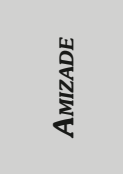 & ن & 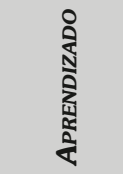 & 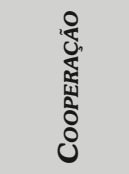 & 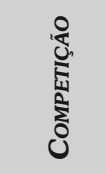 \\
\hline Sírius & $\mathrm{F} 1$ & Sebrae & Sindicato & Polaris \\
\hline Neturno & Sindicato & Sindicato & Sebrae & Neturno \\
\hline Saturno & Sírius & Polaris & $\mathrm{F} 1$ & Bellatrix \\
\hline Polux & Antares & Bellatrix & Polaris & Castor \\
\hline Sindicato & Saturno & Polux & Antares & Numki \\
\hline \multirow[t]{3}{*}{ Antares } & Polux & Antares & Sírius & \\
\hline & Neturno & Sargas & & \\
\hline & & $\mathrm{F} 2$ & & \\
\hline
\end{tabular}

Quadro 1: Comparativo de estrutura central por centralidade indegree das Cinco Forças de adensamento Fonte: Dados primários
O Sebrae e o Sindicato (do setor de calçados e bolsas), tipicamente instituições de apoio a políticas públicas ao setor, são as organizações mais importantes para as relações de fomento a Cooperação e para a disseminação de informações que culminam em Aprendizado para as empresas.

Observa-se no Quadro 1 que as duas empresas mais citadas em relações pessoais (Sírius e Neturno) não estão presentes dentre as organizações que mais ensinam na rede. O fabricante de calçados Polaris, por sua vez, que possui alta capacidade de influenciar as práticas de gestão e o desenvolvimento de novos produtos no setor, é considerada a maior concorrente e uma das empresas que mais cooperam com as demais. Os resultados demonstram ainda que o grupo dos quatro maiores hubs da rede de aprendizagem é composto pelos dois maiores hubs da rede de Cooperação e pelos dois maiores hubs da rede de Competição (Quadro 2). Isso significa que as empresas mais eficientes em obter conhecimento da rede possuem um grande número de parcerias com empresas importantes e percebem alta concorrência local.

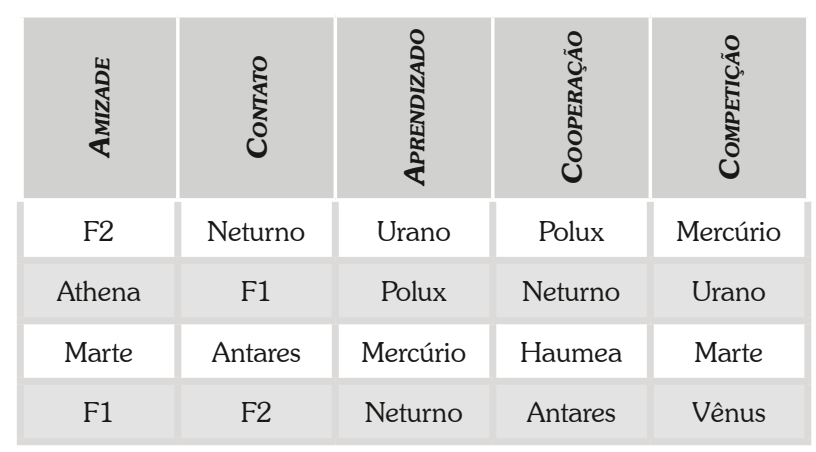

Quadro 2: Comparativo de maiores hubs das Cinco Forças de adensamento

Fonte: Dados primários

\subsection{Discussão dos Resultados}

Ao adaptar o conceito de arranjo produtivo local de Cassiolato e Lastres (2003) para uma perspectiva de redes sociais (GRANOVETTER, 1976), um APL pode ser compreendido como uma rede de relacionamentos interorganizacionais, concentrada geograficamente, delimitada por "nós" inseridos em uma mesma atividade econômica, com múltiplos enlaces, ainda que fracos.

Os membros de um APL podem estar conectados por inúmeros tipos de conexões e fluxos, como 
informação, material, recursos financeiros, serviços e suporte (PROVAN; FISH; SYDOW, 2007). Esses tipos de conexões podem ser compreendidos como forças relacionais que aglutinam constelações (GOMES-CASSERES, 1996) com intensidades e em torno de atores distintos. Nesse sentido, Cinco Forças revelaram-se úteis para a análise de topologias em arranjos produtivos: Amizade, Contato, aprendizagem, Cooperação e Competição.

No sistema de relações concretas (GRANOVETTER, 2005) presentes no APL estudado, representado por sociogramas apresentados no trabalho, as empresas de calçados e bolsas da região metropolitana de Belo Horizonte conduzem suas operações e influenciam-se reciprocamente. Um ponto importante a ser ressaltado é que as informações que fluem pela rede de Contatos não culminam integralmente em criação de conhecimento (enlaces de aprendizagem). Ou seja, a maior parte dos fluxos de informação que circulam na rede não repercute na melhoria da competitividade para o setor - de acordo com a concepção de Huggins (2009) sobre a relação entre Conhecimento e Competitividade.

Em alguns subgrupos da rede, identificou-se a presença de enlaces recíprocos (todos os nós apontam para todos), o que indica maior estabilidade e força do enlace. Nesse sentido, os laços fortes (GRANOVETTER, 1976) que interligam os cliques (EMIRBAYER; GOODWIN, 1994) são identificados apenas nas redes com conteúdo de Amizade e de Contatos. Em relações como de aprendizagem e de Cooperação, altamente relevantes para a melhoria da competitividade do setor, os enlaces revelam-se fracos e mais escassos. A partir desses resultados, verifica-se que em polos produtivos, geograficamente concentrados, Contatos e troca de informações entre os proprietários independem de vínculos formais, contratos, relações comerciais ou relações de dependência. No entanto, a socialização na rede não está diretamente relacionada com benefícios para a empresa e para o setor.

Como concluiu Kraatz (1998) em seu estudo, a estrutura da rede possui um efeito no Aprendizado social. Contudo, tendo em vista a multiplicidade de relações estabelecidas, é importante discernir sobre qual conteúdo dos enlaces é mais determinante neste processo. Nesse sentido, verifica-se que as estruturas das redes de Cooperação e de Competição possuem maior influência sobre o Aprendizado.
A geração de conhecimento relevante advém de relacionamentos quando estes estão atrelados a um processo de desenvolvimento de atividades comerciais ou produtivas conjuntas. Em outras palavras, os relacionamentos são benéficos quando orientados por propósitos claros de Cooperação. A falta de governança em APLs, dessa forma, prejudica o potencial de desenvolvimento coletivo. A alta rivalidade existente no setor também é importante para a aprendizagem, na medida em que enlaces de Competição estimulam práticas de benchmark. Perceber determinada empresa como "maior concorrente" implica investigar constantemente suas inovações em produtos, coleções lançadas, mudanças em processos, relações com fornecedores e com clientes.

O Contato com elos comuns da cadeia produtiva e a observação no mercado fornecem subsídios importantes para que as empresas aprendam com os concorrentes. Como defendem Grimm e Smith (1997), as empresas influenciam-se reciprocamente e contribuem para a melhoria mútua da competitividade. Dessa forma, a alta rivalidade, que por um lado é responsável por ausência de relacionamentos de Cooperação ou por laços fracos, também promove Aprendizado na rede.

Em consonância com os resultados dos trabalhos de Koput e Smith-Doerr (1996), Borgatti e Li (2009) e Giuliane e Pietrobelli (2011) sobre as consequências da conectividade na rede interorganizacional, verificou-se uma correspondência entre a imersão social (MARSDEN, 1981) e o desempenho organizacional. A análise de redes com a utilização da propriedade dos nós "faturamento anual" permitiu a identificação de que todas as empresas com faturamento acima de 1,1 milhão aprendem ou ensinam na rede (estão conectadas a outros nós na rede de aprendizagem). As empresas com desempenho financeiro superior são, portanto, hábeis em absorver informações relevantes sobre o negócio advindas da rede e/ou passíveis de transmitirem conhecimento para a rede.

\section{Considerações Finais}

A importância da estrutura de relacionamentos de um APL encontra-se refletida em sua capacidade para canalizar informações e propiciar conhecimento que pode potencializar ou não projetos coletivos, frente 
aos desafios do ambiente tanto interno quanto externo das firmas.

Ao buscar expandir a ideia de que a estrutura de rede possui um efeito sobre um ganho de desempenho no sistema relacional, este estudo demonstrou constelações formadas a partir do que se decidiu chamar de Cinco Forças relacionais (Amizade, Contato, aprendizagem, Cooperação e Competição) que podem estruturar um Campo de Força estrutural para promover a existência do setor que atuam no plano de negócio com competição e no plano da rede como cooperadores.

Nota-se a falta de estrutura de governança da própria rede, que possa fortalecer os vínculos das cinco forças, como se fosse "cada um cuida do próprio negócio", o que passa uma ideia de alta rivalidade. Assim, é importante que análises comparativas com futuros trabalhos empíricos que explorem qualitativamente o contexto relacional.

A aplicação da ARS para a compreensão dos relacionamentos no APL de calçados e bolsas da RMBH revelou que fatores sociais/Amizade são mais importantes para a densidade e para o volume de interação na rede interorganizacional. Em relação aos fluxos de informações relevantes para a melhoria da Competitividade, infere-se que acompanham, sobretudo, os enlaces de Cooperação e de Competição. São relações mais escassas e mais importantes para o Aprendizado do que densas redes de Amizade.

Sugere-se então que múltiplas forças relacionais, principalmente Cooperação e Competição, moldem o fluxo de aprendizagem na rede e que a topologia de Aprendizado, por sua vez, influencie e seja influenciada por fatores individuais (da empresa) de desempenho. A combinação entre vários conteúdos relacionais e variações individuais proporciona legitimidade para que uma empresa seja seguida $e$ institui mecanismos de transmissão de Aprendizado. Sugere-se ainda, para pesquisas futuras, a utilização das forças relacionais propostas para o dimensionamento de constelações em setores distintos ao calçadista.

Assim, entende-se que as análises de redes APL apresentam-se como método de elevado potencial de contribuição para a área de Estratégia de Redes, particularmente para a compreensão da sua estrutura de competição e cooperação $e$ até recomendações a políticas públicas para incentivo ao setor. A pesquisa ilustra de forma empírica que o paradoxo competição e cooperação coexiste e estabelece um campo de harmônico de solução de convívio na rede em que competição e cooperação coexistem harmonicamente.

\section{REFERÊNCIAS}

AHMADJIAN, C. L. Criação do Conhecimento Interorganizacional: conhecimento e redes. In: TAKEUCHI, H.; NONAKA, I. Gestão do Conhecimento. Porto Alegre: Bookman, 2008.

BARDIN, L. Análise de Conteúdo. Lisboa: Edições 70, 1977. 226 p.

BORGATTI, S. P. NetDraw: Graph Visualization Software. Harvard: Analytic Technologies, 2002.

BORGATTI, S. P.; CARLEY, K.; KRACKHARDT, D. On the Robustness of Centrality Measures under Conditions of Imperfect Data. Social Networks, [S.l.], n. 28 (2), p. 124-136, 2006.

BORGATTI, S. P.; EVERETT, M. G.; FREEMAN, L. C. Ucinet for Windows: software for Social Network Analysis. Harvard, MA: Analytic Technologies, 2002.

BORGATTI, S. P.; LI, X. On Social Network Analysis in a Supply Chain Context. Supply Chain Management, [S.l.], v. 54, n. 2, p. 5-22, 2009.

BRASIL. Ministério do Trabalho e Emprego (MTE). Relação Anual de Informação Social. [S.l.]: RAIS, 2009.

BRASS, D. J. et al. Taking stock of networks and organizations: A multilevel perspective. Academy of Management Journal, [S.1.], n. 47, p. 795-817, 2004.

CASSIOLATO, J.; LASTRES, H. Políticas para promoção de arranjos produtivos e inovativos locais de micro $e$ pequenas empresas: conceito, vantagens e restrições de equívocos usuais. RedeSist, Rio de Janeiro, 2003.

CASTELLS, M. A sociedade em rede: a era da informação, economia, sociedade e cultura. São Paulo: Paz e Terra, 1999.

EMIRBAYER, M.; GOODWIN, J. Network Analysis, Culture, and the Problem of Agency, The American Journal of Sociology, [S.1.], v. 99, n. 6, p. 1.411-1.454, 1994. 
FIEMG. Federação da Indústria do Estado de Minas

Gerais. Perfil das empresas de Calçados e Bolsas da

Região Metropolitana de Belo Horizonte - 2011. Belo Horizonte: FIEMG/IEL, 2011.

FREEMAN, L. C. The Development of Social Network Analysis: a study in the sociology of science. Vancouver: Empirical Press, 2004.

GIULIANE, E. The selective nature of knowledge networks in clusters: evidence from the wine industry. Journal of

Economic Geography, [S.1.], v. 7, p. 139-168, 2007.

GIULIANE, E.; PIETROBELLI, C. Social Network Analysis Methodologies for the Evaluation of Cluster Development Programs. IDB - Inter-American Development Bank, [S.1.], 2011. (Notas Técnicas)

GOMES-CASSERES, B. The alliance revolution: the new shape of business rivalry. Cambridge: Harvard University Press, 1996.

GRANOVETTER, M. The Impact of Social Structure on Economic Outcomes. Journal of Economic Perspectives, [S.1.], v. 19, n. 1, p. 33-50, 2005.

GRANOVETTER, M. Network Sampling: Some First Steps. The American Journal of Sociology, [S.1.], v. 81, n. 6, p. 1287-1303, 1976.

GRIMM, C. M.; SMITH, K. G. Strategy as Action: Industry Rivalry and Coordination. Cincinnati: Thomson South-Western, 1997.

HITT, M. A.; IRELAND, R. D.; HOSKISSON, R. E. Administração estratégica. São Paulo: Thomson Learning, 2003.

HUGGINS, R. Inter-Organizational Networks and Knowledge Flows: a dynamic framework. In: II COPENHAGEN BUSINESS SCHOLL CONFERENCE ON PARTNERSHIPS. Cardiff: Cardiff School of Management, 2009. Anais... Cardiff, 2009.

KRAATZ, M. S. Learning by association? Interorganizational networks and adaptation to environmental change. Academy of Management Journal, [S.1.], v. 41, n. 6, p. 621-643, 1998.
LAZZARINI, S. G.; JOAQUIM, T. A. Z. A formação de constelações: o caso da indústria global de transporte aéreo. Revista de Administração de Empresas, São Paulo, v. 44, n. 2, p. 11-25, 2004.

LIPPARINI, A.; LOMI, A. Interorganizational relations in the Modena biomedical industry: a case study in local economic development. In: GRANDORI, A.

(Ed.). Interfirm networks: organization and industrial competitiveness. Londres: Routledge, 1999.

MARSDEN, P. V. Introducing influence processes into a system of collective decisions. American Journal of Sociology, [S.1.], n. 86, p. 1.203-1.235, 1981.

MARTELETO, R. M. Análise de redes sociais - aplicação nos estudos de transferência da informação, Ciência da Informação, São Paulo, v. 30, n. 1, p. 71-81, 2001.

MARTES, A. C. B. et al. Fórum - Redes Sociais e Interorganizacionais, Revista de Administração de Empresas, São Paulo, v. 46, n. 3, jul. 2006.

MELO, P. T. N. B.; RÉGIS, H. P. Contribuições e Dificuldades na Utilização de Softwares para Análise de Redes Sociais: a produção científica nacional na área de organizações no período de 2001 a 2007. Disponível em: <//users.hotlink.com.br/hregis/Redes/Aula1/IIICBPOT. pdf $>$. Acesso em: 4 abr. 2012

\section{MIGUELETTO, D. C. R. Organizações em rede.}

(Dissertação de Mestrado em Administração Pública). 2001. Escola Brasileira de Administração Pública, FGV. Rio de Janeiro, 2001.

MITCHELL, J. C. Social Networks, Annual Review of Anthropology, [S.1.], v. 3, p. 279-299, 1974.

OLIVER, C. Determinants of Interorganizational Relationships: integration and future directions. Academy of Management Review, [S.1.], v. 15, n. 2, p. 241-265, 1990.

OWEN-SMITH, J.; POWELL, W. W. Networks and Institutions. In: GREENWOOD, R. et al. The Sage Handbook of Organizational Institutionalism. London: Sage Publications, 2007.

POWELL, W. W.; KOPUT, K.; SMITH-DOERR, 1. Interorganizational Collaboration and the Locus of Innovation: Networks of Learning in Biotechnology. Administrative Science Quarterly, [S.1.], v. 41, n. 1, p. 116-145, 1996. 
PROVAN, K. G.; FISH, A. SYDOW, J. Interorganizational Networks at the Network Level: a Review of the Empirical Literature on Whole Networks. Journal of Management, [S.l.], v. 33, n. 3, p. 520-626, 2007.

RECUERO, R. C. Teoria das Redes e Redes Sociais na Internet: Considerações sobre o Orkut, os Weblogs e os Fotologs. In: ENCONTRO DOS NÚCLEOS DE PESQUISA DA XXVII INTERCOM, 2004, Porto Alegre.

Anais... Porto Alegre: Intercom, 2004.

SCOTT, J. Social Network Analysis: a handbook. GraBretanha: Sage, 1991.

SOUZA, Q.; QUANDT, C. O. Metodologia de Análise de Redes Sociais. In: DUARTE, F; QUANDT, C.; SOUZA, Q. (Org.). O Tempo das Redes. São Paulo: Perspectiva, 2008. p. 31-63.

TAKEUCHI, H.; NONAKA, I. Gestão do conhecimento. Porto Alegre: Bookman, 2008.

TSAI, W. Knowledge Transfer in Intraorganizational Networks: Effects of Network Position and Absorptive Capacity on Business Unit Innovation and Performance. Academy of Management Review, [S.1.], v. 44, n. 5, p. 996-1.004, 2001.

VENKATRAMAN, N.; LEE, C. Preferential linkage and network evolution: A conceptual model and empirical test in the U.S. video game sector. Academy of Management Journal, [S.1.], v. 47, n. 6, p. 876-888, 2004.

WIT, B.; MEYER, R. Strategy: process, content, context. 4. ed. Reino Unido: Cengage Learning, 2010. 